Physics, Chemistry, and Dynamics of Interplanetary Dust

ASP Conference Series, Vol. 104, 1996

Bo A. S. Gustafson and Martha S. Hanner (eds.)

\title{
Meteorites from Comets? Recent Observational Developments
}

\author{
Humberto Campins \\ Department of Astronomy University of Florida Gainesville, Florida \\ 32611
}

\begin{abstract}
Although most meteorites come from asteroids, some could originate from short-period comets. The identification of comet-asteroid transition object 4015 Wilson-Harrington as the first Apollo object known to have a cometary origin, confirms that short-period comets leave behind nonvolatile residues, from which meteoroids with low Earth-encounter velocities can originate. Studies of cometary fireballs suggest that some cometary meteoroids have high enough strengths to have survived atmospheric entry as cometary meteorites, which have either not been found or recogized. The presence of unequilibrated anhydrous silicates in cometary dust represents a significant difference between cometary material and CI and CM meteorites.
\end{abstract}

\section{Introduction}

Recent developments, namely the identification of comet-asteroid transition object 4015 Wilson-Harrington, and the detection of anhydrous silicates in four comets, are relevant to the topic of cometary meteorites. In this paper the question of whether comets can yield meteorites is revisited, and two main conclusions are reached. First, short-period comets do leave behind nonvolatile residues from which meteoroids, capable of reaching the Earth surface as meteorites, can originate. Second, the occurrence of unequilibrated anhydrous silicates in cometary dust represents a significant difference between one type of cometary material and CI and CM meteorites. These anhydrous silicates might help us identify cometary meteorites.

The possible cometary origin of some meteorites has been discussed extensively (e.g., Wetherill 1974, 1978). However, a number of arguments suggest that none of the meteorites studied so far are cometary ${ }^{1}$ Can we, therefore, rule out comets as a plausible source for some, as yet undetected meteorite? The answer is NO! In the following two sections arguments in support of this conclusion are discussed.

${ }^{1}$ a) Anders (1986 and references therein) concluded from studies of trapped solar wind noble gas, that most stony meteorites must come from the regoliths of objects in or near the asteroid belt and not from comets. b) Lipchutz et al. (1989) argue that there are significant differences in the meteoritic and cometary dust composition. For example, the bulk carbon and nitrogen contents in Comet Halley's dust is significantly higher than in the known chondrites (Brownlee et al. 1987). 


\section{Short-Period Comets}

Several arguments support the notion that short-period comets leave behind nonvolatile residues, from which meteoroids with low Earth-encounter velocities could originate. Furthermore, these cometary meteoroids appear strong enough to survive atmospheric entry.

A. Wetherill $(1974,1978)$ summarized a number of arguments suggesting that if cometary meteorites exist, they are most likely delivered from the nonvolatile residue of short-period comets. Two of the main predictions reviewed by Wetherill (1978) have been confirmed. First, that nonvolatile residues of comets do exist, and second, that some of the Apollo objects (Earth-crossing asteroids) are extinct or dormant comets. Observations of the nuclei of three low activity comets, Arend-Rigaux (Millis et al. 1988), Neujmin 1 (Campins et al. 1987), and Tempel 2 (A'Hearn et al. 1989), showed that the active fraction of their nuclear surface was less than $1 \%$ in all three cases. Furthermore, 3200 Phaethon has been identified as the parent body of the Geminid meteor shower, and subsequently there have been a number of arguments in favor of the cometary origin of this object (e.g., Weissmann et al. 1989. and references therein). These results indicate that comets can leave behind a sizeable nonvolatile residue when their activity ceases. Moreover, the identification of 40151979 VA as Comet Wilson-Harrington 1949 III (Bowell 1992, Marsden 1992), confirms that some fraction of near-Earth asteroids have a cometary origin. This is the first Apollo object known to have a cometary origin, and it has received the unusual designation of 4015 Wilson-Harrington (henceforth $4015 \mathrm{~W}-\mathrm{H}$ ). Orbital characteristics make $4015 \mathrm{~W}-\mathrm{H}$ particularly interesting even among the comet-asteroid transition objects. It has a low inclination orbit ( 2.6 degrees), perihelion just inside Earth's orbit (0.995AU), and aphelion in the asteroid belt (4.3AU). This orbit has the potential of delivering meteoroids to Earth at lower velocities than most other near-Earth objects; hence, $4015 \mathrm{~W}-\mathrm{H}$ is potentially a meteorite producing comet (Campins et al. 1995). In fact, $4015 \mathrm{~W}-\mathrm{H}$ may be the parent body of one of the fireballs that Halliday et al. (1989) estimate have dropped meteorites with recoverable masses. The dynamical arguments linking $4015 \mathrm{~W}-\mathrm{H}$ with this fireball are discussed in a paper by Gustafson et al. (1996).

B. An analysis of fireballs believed to be of cometary origin (those with retrograde orbits with aphelia beyond Jupiter, and those in meteor streams) showed that most of these fireball objects, although weaker than most recovered meteorites, have the potential to yield a significant amount of meteoritic material (Wetherill and ReVelle 1982) ${ }^{2}$. This conclusion is strengthened by the findings of Babadzhanov (1992). He argues that the densities of many shower meteors have been underestimated, and can be as high as carbonaceous chondrites or even ordinary chondrites. In other words, some cometary fireballs have low enough atmospheric entry velocities and high enough cohesive strengths to have produced cometary meteorites, which have either not been found or recognized.

\footnotetext{
${ }^{2}$ One argument often given against meteorites from comets, is the lack of falls during showers (except for one coincidental iron meteorite fall). This argument is not valid because the entry velocities of most shower meteors is sufficiently high $(>25 \mathrm{~km} / \mathrm{sec})$ that even strong achondrite meteorites would not survive atmospheric entry.
} 


\section{Unequilibrated Anhydrous Silicates}

Observations of anhydrous silicates in four comets, and in interplanetary dust particles (IDPs) of "cometary" origin (see item C below) constitute new compositional evidence that might allow us to identify cometary meteorites.

\section{A. Ground based observations of crystalline olivine in Comet Hal-} ley and three other comets. Silicate emission in the 8-13 micron spectral region has been observed in comets since 1970. However, the first detection of an emission peak near 11.25 microns was made in Comet Halley (Bregman et al. 1987, Campins and Ryan 1989), and was identified with crystalline olivine similar to that found in some IDPs. Crystalline olivine has now been identified in three other comets (Hanner et al. 1994 and references therein). Other silicates, most likely glassy pyroxene and olivine (but not hydrated silicates), are necessary to explain the rest of the 8-13 micron emission.

B. Spacecraft mass spectra of dust in Comet Halley. In remarkable agreement with the infrared observations described above, the impact dust analyzers in the Giotto and Vega spacecraft indicate that silicates in Halley's dust are anhydrous. The observed distribution of $\mathrm{Fe} /(\mathrm{Fe}+\mathrm{Mg})$ in Halley's dust matches best the distribution observed in IDPs dominated by anhydrous minerals, and is unlike the distribution observed in carbonaceous chondrite matrix (e.g., Brownlee et al. 1987, Bradley 1988, Lawler et al 1989).

C. Analogy with IDPs. It is significant that the IDPs matching the distribution of $\mathrm{Fe} /(\mathrm{Fe}+\mathrm{Mg})$ in Halley's dust are not only anhydrous, but are also those identified as most likely to be cometary based on other dynamical and physical characteristics (such as their atmospheric entry speed and their infrared spetrum. Sandford and Bradley 1989). Those IDPs classified as most likely to be asteroidal are generally hydrated and show a distribution of $\mathrm{Fe} /(\mathrm{Fe}+\mathrm{Mg})$ different to that in Comet Halley dust, but similar to carbonaceous chondrite matrix.

The presence of anhydrous silicates in comets is unexpected for many researchers. For example, CI and CM chondrites have been proposed as the most likely meteorites to have a cometary origin because their elemental composition approximately matches that of comets (e.g., Wasson 1985). However, this view is based in part on the higher volatile contents of these meteorites being interpreted as indicative of more primitive material. It is now evident that the higher volatile content in CI and CM is the result of aqueous alteration (e.g.. Zolensky and McSween 1989). In the case of the anhydrous cometary silicates, it appears that the water and at least some silicates coexist in an environment that does not favor hydration (McSween and Weissman 1989). In fact, Comet Halley's dust, as well as most IDPs (particularly the "cometary" IDPs) are highly unequilibrated mineralogically, chemically and isotopically, such that water ice and silicates are only two of many unequilibrated phases we expect to find in comets. At this point it must be emphasized that the available observations do not rule out the existence of some hydrated silicates in comets (e.g., Rietmeijer and McKinnon 1985); nevertheless, anhydrous silicates appear to dominate in the comets observed so far.

In summary, short-period comets leave behind nonvolatile residues such as $4015 \mathrm{~W}-\mathrm{H}$. Such objects appear to be capable of producing meteoroids that have low enough atmospheric entry velocities and high enough strengths to have 
produced cometary meteorites. These meteorites have either not been found or not been recognized. The presence of anhydrous silicates with a distribution of $\mathrm{Fe} /(\mathrm{Fe}+\mathrm{Mg})$ like that in Comet Halley's dust might help us to identify cometary meteorites. Although CI and CM chondrites have elemental compositions similar to comets, cometary dust contains unequilibrated anhydrous silicates that are significantly different from those found in CI and CM meteorites. However, the available observations do not rule out the existence of some hydrated silicates in comets. Acknowledgments. I thank Beth Clark, Dave Kring, Scott Sandford and Tim Swindle for useful discussions. This work was supported by the National Science Foundation.

\section{References}

A'Hearn,M.F., Campins,H., Schleicher,D.G and Millis,R.L. 1989, ApJ,347,1155 Anders,E. 1986, ESA SP 249, 31

Babadzhanov,P.B 1992, In Asteroids Comets and Meteors 1991, LPI pub., 23

Bowell,E. 1992, IAU Circ. 5585

Bradley,J. P 1988, Geochim. Cosmochim. Acta, 52, 889

Brownlee,D. E., Wheelock, M.M., Temple, S., Bradley, J. P., and Kissel 1987, Lunar and Planet. Sci. Conf. 13, 133

Bregman et al. 1987, Astron. Astrophys. 187, 616

Campins,H. A'Hearn,M.F.and McFadden,L.A. 1987, ApJ.316, 847

Campins,H. and Ryan, E. V. 1989, ApJ. 341, 1059

Campins,H., Osip,D.J., Rieke,G.H. and Rieke,M.J. 1995,Plan. Sp. Sci. 43,733

Gustafson,B. Adolfsson,L. and Campins,H. 1996, to be submitted to Icarus.

Halliday,I., Blackwell,A.T., and Griffin,A.A. 1989, J.Roy.Ast.Soc.Can. 83,49

Hanner,M.S., Hackwell,J.A., Russell,R.W., Lynch,D.K. 1994, Icarus, 112,490

Lawler,M.E., Brownlee,D.E., Temple,S.,and Wheelock,M.M.1989,Icarus,80,225

Lipchutz,M.E., Gaffey, M.J., and Pellas, P. 1989, In Asteroids II, 740

Marsden,B. 1992, IAU Circ. 5585 and 5586

McSween,H.Y., and Weissman,P.R. 1989, Geoch. Cosmoch. Acta, 53, 3263

Millis,R.L., A'Hearn,M.F and Campins,H. 1988, ApJ, 324, 1194

Rietmeijer.F.J.M., and McKinnon,I.D.R. 1985, J. Geophys. Res. 90, D149

Sandford.S.A. and Bradley,J.P. 1989, Icarus, 82, 146

Wetherill,G. W 1974, Ann. Rev. Earth Planet. Sci. 2, 303

Wetherill,G.W. 1978, In Asteroids, NASA CP 2053, 17

Wetherill,G.W and ReVelle,D.O. 1982, In Comets, U. of Arizona press, 131

Wasson,J.T., 1985, Meteorites, W.H. Freeman publisher.

Weissman,P.R.. A'Hearn,M.F., McFadden,L.A., and Rickman,H. 1989. In Asteroids $I I$. eds. Binzel et al., U of Arizona press, 880

Zolensky.M., and and McSween,H.Y 1989, In Meteorites and the Early Solar System, eds. Kerridge and Matthews, U. of Arizona press, 114 